\title{
A case of complication after a degloving operation of melanoma of the penis-repairing urethrocutaneous fistula with a pedicled gracilis flap
}

\author{
Hin-Lun Liu • Jimmy Yu-Wai Chan
}

Received: 29 April 2010 /Accepted: 29 August 2010/Published online: 28 September 2010

(C) The Author(s) 2010. This article is published with open access at Springerlink.com

\section{Introduction}

Melanoma of the penis is a rare clinical condition. In this case report, our patient had a wide excision for melanoma of the penile skin. After the operation, he developed a urethrocutaneous fistula at the bulbar urethra. The defect was then repaired successfully with a pedicled gracilis flap. The surgical treatment of melanoma of the penis and various reconstructive options for the iatrogenic urethrocutaneous fistula will be discussed.

\section{Case report}

A 38-year-old man developed a subdermal nodule on the penile shaft. Excisional biopsy was performed by a private doctor; this confirmed melanoma with involvement of the resection margin. He was then referred to us for further management. On examination, there were multiple subdermal nodules on the penile shaft; these were freely mobile from the underlying Bucks fascia. The overlying epidermis was intact. The glans and urethra were not involved (Fig 1). There were no palpable groin lymph nodes. The clinical diagnosis was

H.-L. Liu $(\bowtie) \cdot$ J. Y.-W. Chan

Division of Plastic and Reconstructive Surgery,

Department of Surgery, University of Hong Kong Medical Centre,

Queen Mary Hospital,

Hong Kong, SAR, China

e-mail: lawrencehlliu@gmail.com melanoma of the penile skin with subdermal metastases. The need for operation to obtain local control of the disease was explained to the patient. He refused radical resection of the penis and opted for a more conservative operation.

Wide excision of the penile and scrotal skin was performed. The superficial fascia of the penis was taken as the deep resection margin; Bucks fascia was not breached since it was not involved by tumour. The testes were buried within the residual scrotal skin. The denuded penile shaft was then skin-grafted (Fig. 2). Sentinel lymph node sampling was performed. A urinary catheter was inserted at the end of operation.

The skin graft was healthy on the first few days after operation. The urinary catheter was removed on postoperation day 6 , but a urethrocutaneous fistula was noted at the region of the bulbar urethra (Fig. 3). The histological examination confirmed the diagnosis of melanoma for all subdermal nodules; the maximum tumour thickness was $13 \mathrm{~mm}$. Both radial and deep resection margins were clear. The sentinel lymph node at the left groin showed metastatic melanoma. Repair of the urethrocutaneous fistula and left groin dissection were therefore performed.

A pedicled gracilis muscle flap based on the terminal branch of the adductor artery was raised. Both the muscle origin and insertion were detached to improve the arc of rotation. The anterior branch of the obturator nerve was transected deliberately to prevent muscle contraction and promote subsequent muscle atrophy. The muscle was transferred to the defect through the subcutaneous tunnel. A buccal mucosal graft was harvested and used for inner lining (Fig. 4). The external 


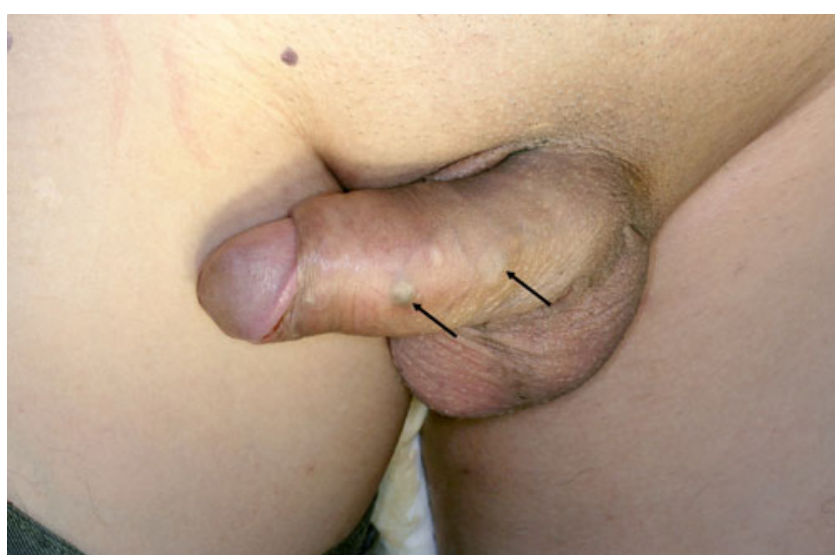

Fig. 1 Multiple subdermal nodules at the penile shaft (arrows). The overlying epidermis was intact

aspect of gracilis muscle was skin-grafted (Fig. 5). A groin dissection was also performed in the same procedure.

A urinary catheter was kept in situ for 3 weeks before a contrast cystogram was performed. The antegrade cystogram showed relative narrowing of the bulbar urethra; otherwise, there was no leakage of contrast (Fig 6). The patient refused adjuvant therapy and opted for expectant management. There was no local recurrence at 4 months after operation.

\section{Discussion}

Melanoma of the penis is a rare clinical condition which has been presented previously only in case reports and short

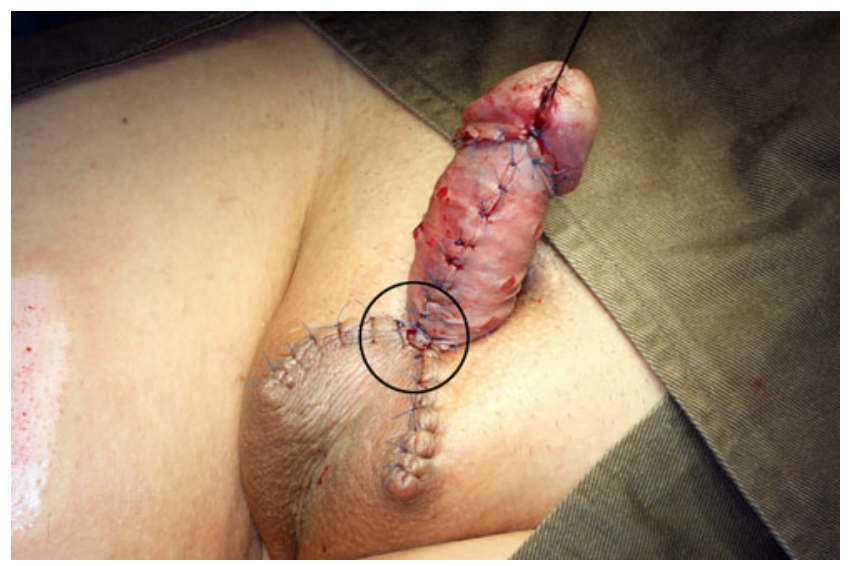

Fig. 2 After wide excision of the penile and scrotal skin, the penile shaft was skin-grafted. The scrotal skin was sutured to the corpus spongiosum. We postulated that the cutting through of stitches at this region (circle) was the cause of urethra injury

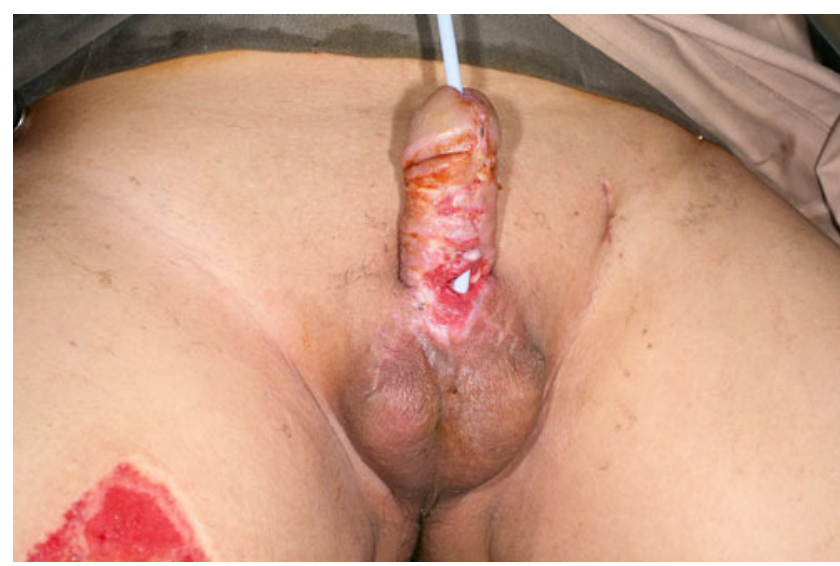

Fig. 3 The urethrocutaneous fistula at the region of bulbar urethra

series [1-4]. The mainstay of treatment is surgical. For lesions at the glans or urethra, penectomy is usually required for disease control. For lesions arising from the penile skin, the extent of operation should be judged by the tumour thickness. In the largest series to date, SánchezOrtiz et al. found that wide excision without penectomy can provide effective local control for low-stage (i.e. T1, T2) penile melanoma. On the other hand, for thick tumour (i.e. T3, T4), there is still a lack of evidence on the optimal surgical treatment for local control [3]. In our case, as the glans and urethra were not involved and there was no deep involvement of the tumour, wide excision of penile skin was performed for local control.

After the degloving operation of the penis, the structures left behind were the corpora cavernosa and the corpus spongiosum. The penile urethra, particularly the bulbar

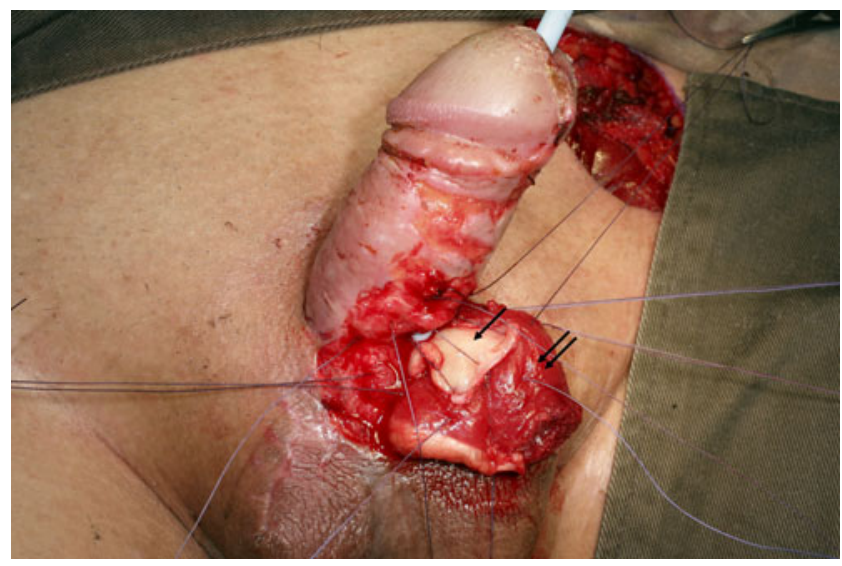

Fig. 4 The fistula was closed in two layers. The inner mucosal layerbuccal mucosal graft (single arrow); the outer layer-pedicled gracilis muscle flap (double arrow) 


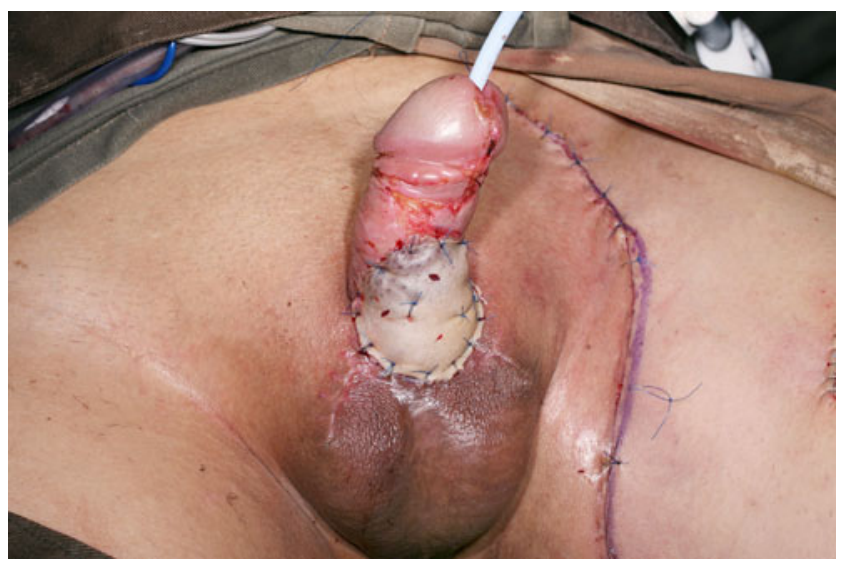

Fig. 5 The external surface of the gracilis muscle was skin-grafted

part, is situated superficially in the corpus spongiosum. The bulbar urethra is therefore the most vulnerable part of the penile urethra. In our case, we postulated that the development of urethrocutaneous fistula is due to the cutting through of stitches at this region. After the excision of the penile and scrotal skin, the residual scrotal skin was sutured to the corpus spongiosum to assist wound closure. As the corpus spongiosum is not a strong structure that can hold stitches firmly, together with the pulling force conferred by the erection of penis, the stitches placed at this region cut through easily. This caused the injury to the underlying bulbar urethra and the subsequent fistula formation. To avoid this complication, a tension-less wound closure should be adopted at this region.

The management of an urethrocutaneous fistula in the setting of denuded penis is not well documented in the

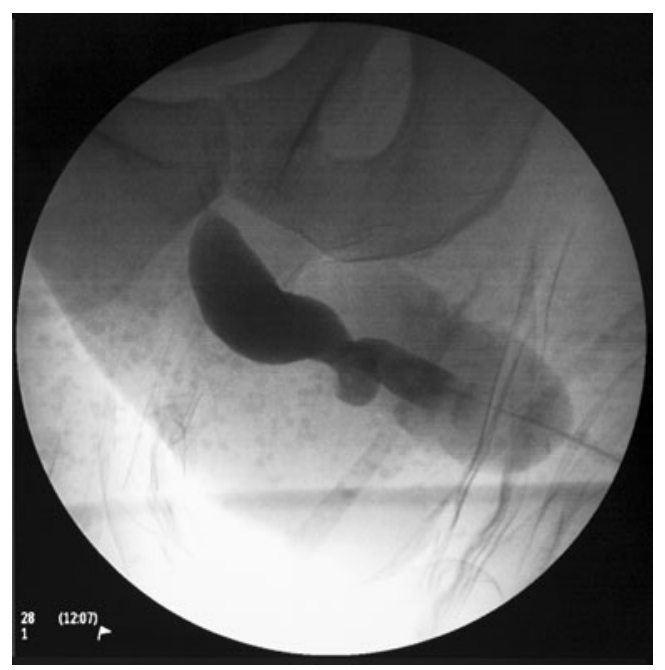

Fig. 6 The antegrade cystogram showed relative narrowing of the bulbar urethra. There was no contrast leakage literature. This condition excludes the use of local skin or fascial flaps; therefore, the reconstructive methods left available are regional flaps. Heckler et al. have described the use of gracilis and inferior-based rectus abdominis muscle and myocutaneous flaps for the repair of posttraumatic and iatrogenic urethrocutaneous fistula [5]. Apart from the above reconstructive methods, other feasible options include groin and thigh flaps. Although rectus abdominis muscle flap can provide a good bulk of vascularized tissue for repair, it was not used because of the debilitating donor site morbidity. Groin and thigh flaps were not used in this case because a groin dissection had been performed on one side, and it was preferred not to disrupt the groin region on the nonoperated side. In our opinion, the use of the gracilis muscle flap not only provides vascularized tissue for repair, a good aesthetic outcome can also be achieved. The thin sheet of gracilis muscle with the subsequent denervation atrophy helps the flap to blend well with the surrounding tissue which provides a good aesthetic outcome (Fig. 7). In addition, the harvest of the gracilis muscle causes only minimal morbidity, as the powerful adductor muscles can compensate for the functional loss.

In conclusion, melanoma of the penis is a rare condition. Surgery is the mainstay of treatment. During a degloving operation of the penis, a tension-less closure should be provided at the region of the bulbar urethra to avoid urethral injury. The development of urethrocutaneous fistula in the setting of a denuded penis is a challenge in reconstructive surgery. The pedicled gracilis flap is a feasible reconstructive option which can successfully repair the fistula and achieve a good aesthetic result.

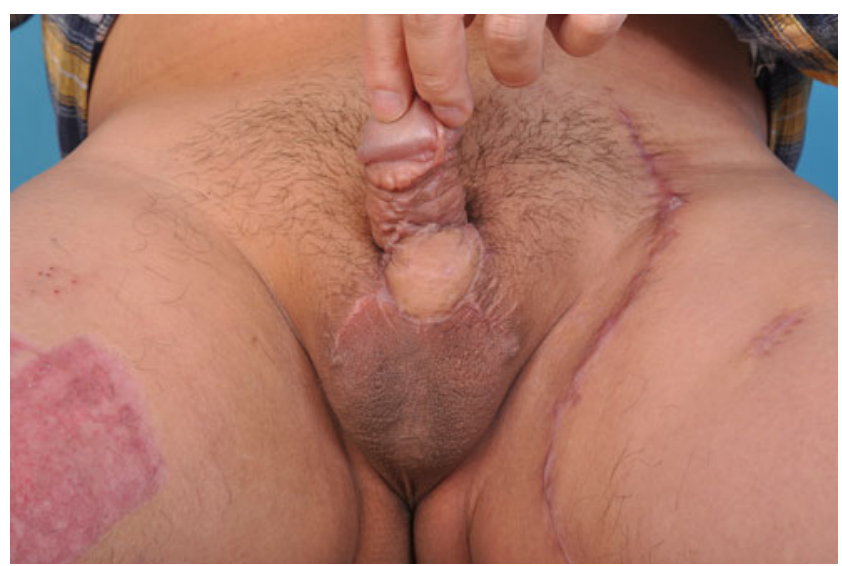

Fig. 7 The urinary catheter was removed after the cystogram. The flap blended nicely with the surrounding tissue 
Open Access This article is distributed under the terms of the Creative Commons Attribution Noncommercial License which permits any noncommercial use, distribution, and reproduction in any medium, provided the original author(s) and source are credited.

\section{References}

1. Kim S, Brock GB, Temple CL (2007) Minimizing morbidity in the treatment of penile melanoma: Mohs micrographic surgery and sentinel lymph node biopsy. Can J Urol 14:3467-3470
2. Te CC, Vemulapalli S, Confer SD, Culkin DJ (2008) Recurrent malignant melanoma of the penis. Urology 72(1185):e15e16

3. Sánchez-Ortiz R, Huang SF, Tamboli P, Prieto VG, Hester G, Pettaway CA (2005) Melanoma of the penis, scrotum and male urethra: a 40-year single institution experience. J Urol 173:19581965

4. Oldbring J, Mikulowski P (1987) Malignant melanoma of the penis and male urethra. Cancer 59:581-587

5. Heckler FR, Aldridge JE Jr, Songcharoen S, Jabaley ME (1980) Muscle flaps and musculocutaneous flaps in the repair of urinary fistulas. Plast Reconstr Surg 66:94-101 